\title{
Diseño y ensayo de un secador solar para madera
}

\author{
Enrique Martínez-Pinillos Cueto ${ }^{1}$
}

\begin{abstract}
RESUMEN
Uno de los principales problemas que enfrenta la industria de la madera, es el uso de la madera con un contenido de humedad apropiado. Este problema se presenta desde la fabricación y durante la vida en servicio del producto. Con el fin de contribuir a resolver este problema, en este articulo se presenta el diseño de una estufa de secado solar para madera, de 1200 pies/tabla de capacidad, la cual se construyó en el área del Departamento de Productos Forestales y Conservación de Bosques. En este trabajo se explica la metodología del diseño, la operación de la secadora y los pasos para controlar la temperatura y la humedad durante el secado. A la fecha, se pueden ofrecer resultados de cuatro cargas de secado, sobre todo en cuanto a tiempo de secado, contenido de humedad inicial y final, temperatura y humedad relativa registrada dentro y fuera de la estufa, así como también la relación entre la temperatura y las horas diarias de insolación. Esta estufa es una opción interesante para los que trabajan la madera, debido a que su construcción no requiere materiales caros y que son fáciles de obtener; asimismo, su construcción la puede hacer una persona con mediano entrenamiento.
\end{abstract}

PALABRAS CLAVE:

Secado solar, tiempo de secado, diseño de secador, Pinus spp, Hevea brasiliensis, operación del secador.

\begin{abstract}
The utilization of wood with an appropriate moisture content is one of the main problems that faces wood industry. This problem begins since the fabrication phase and remains during its service life. In order to contribute to the solution of this trouble, this paper presents the design of a solar kiln for drying wood, of 1200 board feet capacity, built in the facilities of the Department of Forest Products and Forest Management. It explains the methodology of the design, its operation, the wood piling and the steps for controlling temperature and humidity while drying. To this day, results can be given for four drying loads, particulary about drying time, initial and final moisture content, temperature and relative humidity records, inside and outside the oven, as well as the relation between temperature and the daily insolation hours. This kiln is an interesting option for wood manufacturers, because its construction requires inexpensive materials easy to obtain; also, the construction can be made by medium qualified woodworkers.
\end{abstract}

KEY WORDS:

Solar kiln drying, drying time kiln, design, Pinusspp, Hevea brasiliensis, kiln operation.

1 Departamento de Productos Forestales y Conservación de Bosques, Instituto de Ecología, A. C., Apdo. Postal 63, 91000 Xalapa, Ver. México.

Manuscrito recibido para su publicación el 30 de Septiembre de 1996 


\section{INTRODUCCION}

Uno de los principales problemas que tiene el sector de transformación de la madera, es obtenerla con valores de contenido de humedad apropiado para la fabricación de productos, en los que se usa este material como materia prima, ya que si se elaboran los productos con madera que tenga un elevado contenido de humedad, en el transcurso de los días posteriores a su elaboración la madera perderá poco a poco el agua que se encuentra en su interior, hasta lograr un equilibrio con el medio ambiente, ocasionando en principio, que las dimensiones de las partes que forman los objetos disminuyan y esto a su vez ocasionará algunos otros problemas que pueden manifestarse como fallas en las uniones, baja resistencia en las líneas de pegamento, desprendimiento de los acabados, grietas, etc. Como se ve, es indispensable usar madera seca para poder fabricar productos de buena calidad. Para secar la madera existen varios métodos, que van desde el secado al aire libre, el cual no requiere ningún tipo de equipo, pues sólo se necesita un área adecuada para apilar madera, un techo y una buena orientación de las pilas de secado (Rodríguez, 1988), hasta otros más sofisticados que requieren equipo especializado, como son el secado al alto vacío y con ultrasonido.

El secado solar es un proceso que se ha utilizado en distintas partes del mundo, pero principalmente en Asia y Africa (Milota y Wengert, 1995), esta tecnología se puede considerar como intermedia, entre los dos métodos utilizados con mayor frecuencia entre el sector de transformación de la madera de nuestro país, que son el secado al aire libre y el secado convencional, el cual usa como fuente generadora de calor serpentines con vapor de agua en su interior. Con el uso de este método se puede llevar un control preciso sobre el proceso de secado; en México existen guías de secado para las especies más comerciales como las mostradas por Fuentes et al., 1996. La desventaja que tiene este proceso es el costo de adquisición del equipo. Con el secado solar se pueden alcanzar contenidos de humedad finales de la madera con valores similares a los obtenidos en el secado convencional, aún cuando se emplee mayor tiempo de secado, sin embargo el tipo de equipo que se necesita para poner en marcha este proceso tecnológico es comercial y se puede conseguir fácilmente, con lo que se facilita la construcción de este tipo de secador.

La fuente generadora de calor para hacer funcionar esta tecnología es el sol, que es la estrella más cercana a la tierra y la más importante, pues sin ella la vida no existiría en nuestro planeta. Usando esta fuente de energía se disminuye el costo en los procesos de trasformación, debido a que del porcentaje total de la energía que se emplea para manufacturar madera, el secado consume entre el 60 y $70 \%$ (Helmer, 1986).

La energía que el sol produce e irradia se origina mediante reacciones nucleares que ininterrumpidamente se realizan en su interior; esta energía se presenta en la tierra como radiación solar y tarda ocho minutos en llegar a la superficie terrestre (Censolar, 1990). La situación geográfica de nuestro país, la hace ideal para colocar colectores solares, ya que incide sobre todas sus latitudes obteniendo buen rendimiento en ellos. Los secadores solares pueden dividirse en dos tipos: modelos con colectores solares incluidos en la estructura del producto y modelos en los que el colector se encuentra en un lado de la cámara de secado (Olivera et al., 1982) y estos a su vez se pueden clasificar en tres tipos: equipos con circulación de aire natural, con circulación forzada mediante ventiladores y con circulación forzada y controles automáticos (Simpson, 1992); a su vez, cada una de estas posibilidades se 
puede realizar completamente fijada al sitio donde funcionará o con la característica de ser portátil.

\section{OBJETIVO}

El objetivo de este trabajo fue diseñar, construir, y probar con diferentes corridas de secado un prototipo de secador solar para madera, tomando como base la información recopilada, y los resultados obtenidos en modelos diseñados con anterioridad en el Departamento de Productos Forestales y Conservación de Bosques.

\section{METODOLOGIA DE DISEÑO}

Para desarrollar el diseño del secador solar se partió de la recopilación de literatura relacionada con el tema, realizando un análisis de sus características, para poder conocer las ventajas y desventajas que pueden presentar y tener así un buen punto de partida para elaborar el diseño del producto (Jones, 1983). Otra información que se tomó como base para este diseño, fue la que se obtuvo en la realización y puesta en operación de prototipos de secadores solares realizados con anterioridad, entre los que resaltan, el construido en la ciudad de Xalapa, Ver., con una capacidad de 1,000 pies/tabla, el que se instaló en empresa Etla en la ciudad de Oaxaca, con una capacidad de 3,000 pies/tabla y el construido en la empresa Duraplay de Parral en Chihuahua, con una capacidad de 6,000 pies/tabla aproximadamente, mismo que se usó como presecador antes de introducir la madera a las estufas del tipo convencional con que cuenta la empresa.

Después del análisis de la información recabada, se ensayaron posibles soluciones al problema, siendo evaluadas detalladamente para seleccionar de entre ellas la que presentara mejores posibilidades de éxito. Cuando se seleccionó la más viable, se procedió a desarrollar el diseño con detalle para poder llegar a elaborar los planos constructivos que permitieran la fabricación del prototipo de secador solar (Fig. 1).

Las partes que forman el secador solar diseñado son: colector solar para el calentamiento de aire, ventiladores para mover el aire caliente, área para colocar la madera durante el proceso, ventilas para salida de aire, y puerta de acceso para la madera (Fig. 2). El colector es el encargado de captar la radiación solar y calentar el aire que circula en su interior, en este caso está construido en la parte superior de la estructura de la estufa, convirtiéndose en el techo de la misma; está formado por una lamina galvanizada pintada de color negro mate, que funciona como cuerpo absorbedor de la radiación solar. En la parte superior de la lámina se coloca una capa de vidrio, que es la encargada de realizar junto con la lámina el efecto invernadero, con el cual se genera una cámara de aire caliente entre la lámina y el vidrio. La principal ventaja que se obtiene con este modelo de colector es que, al colocar en la parte inferior de la lámina una capa de madera, se está obteniendo una segunda cámara de calentamiento de aire (Fig. 3), con lo que se mejora la eficiencia del colector con relación a los modelos diseñados con anterioridad. La inclinación que tiene el colector es de $20^{\circ}$, que corresponde a la latitud del lugar en donde se encuentra instalado el prototipo. Para fines prácticos, se puede decir que la inclinación del colector podrá variar $5^{\circ} \sin$ afectar considerablemente su eficiencia. La parte más alta del colector se coloca orientada hacia el norte, para aprovechar al máximo los rayos solares durante todo el día (Censolar, 1990). En el 


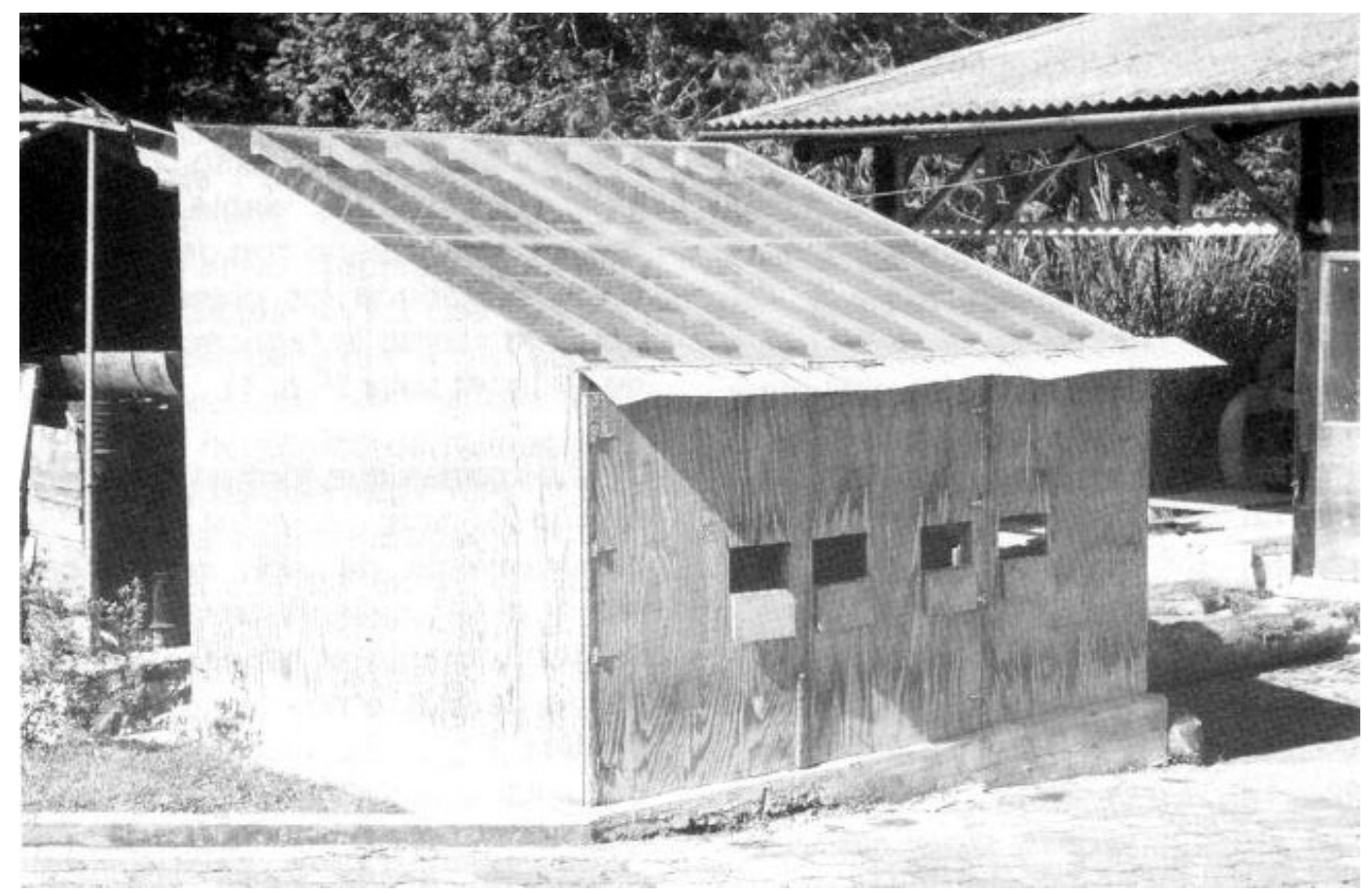

Figura 1. Prototipo de secador solar para madera

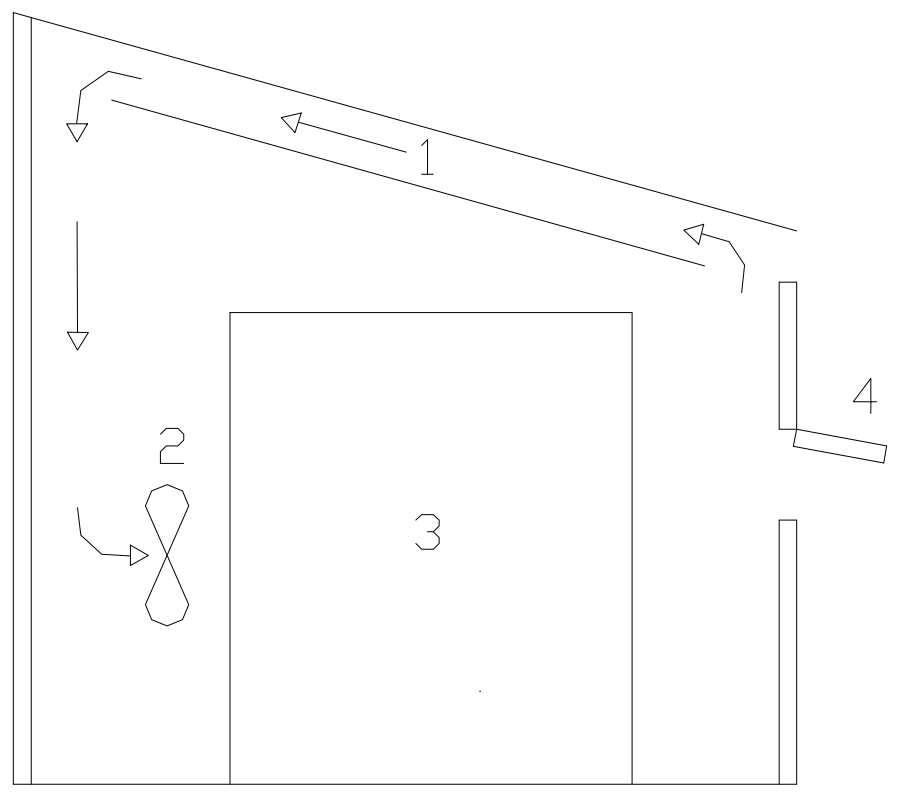

Figura 2. Partes del secador solar para madera: 1) colector; 2) ventiladores; 3) área para secado; 4) ventila para extracción de aire húmedo 


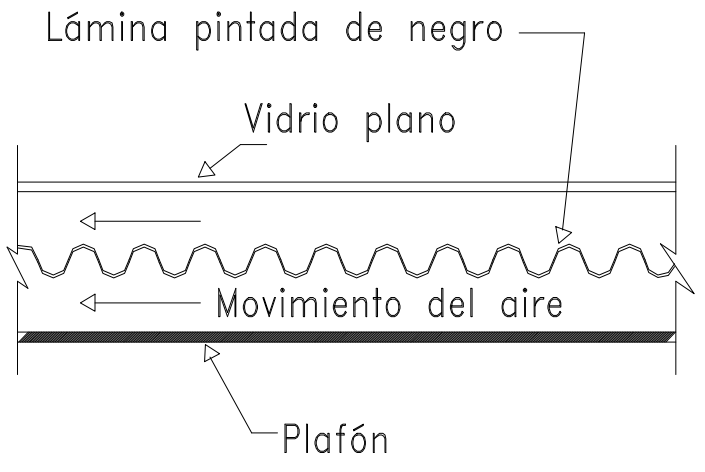

Figura 3. Vista del colector solar

interior de la cámara de secado, en la parte superior de la pared que se orientó hacia el norte se colocan dos ventiladores con aspas de $60 \mathrm{~cm}$ de diámetro y motor con capacidad de 1/2 caballo de fuerza, para extraer el aire que se calienta dentro del colector y posteriormente pasarlo a la cámara de secado en donde se distribuirá a través de la madera.

La estructura del secador y las puertas de acceso están fabricadas con madera sólida, de sección de 38 × 87 mm (2 x 4 plg) y unidas con clavos. Como acabado se le colocó un recubrimiento de madera contrachapada de $16 \mathrm{~mm}$ de grosor, resistente a la humedad. Las dimensiones en planta del secador son: $3.66 \mathrm{~m}$ de largo por $3.05 \mathrm{~m}$ de ancho y $2.90 \mathrm{~m}$ en la parte más alta, que corresponde a la pared norte y $1.80 \mathrm{~m}$ en la parte más baja que corresponde a la pared sur, en donde se encuentran localizadas las puertas de acceso. El piso del secador es una plancha de concreto, a la que se encuentra anclada la estructura. En la pared orientada hacia el sur, se colocaron cuatro ventilas con dimensiones de $40 \mathrm{~cm}$ de largo y $20 \mathrm{~cm}$ de ancho, para poder controlar la humedad y la temperatura en el interior, las que deben situarse en la posición más favorable para la extracción del aire, cuando se requiera (Steinmannn, 1989).

La fabricación con madera, de la estructura del secador, representa una ventaja en cuanto al ahorro de mano de obra durante el proceso constructivo ya que la construcción puede llevarla a cabo el mismo usuario del secador, quien normalmente tiene experiencia en el trabajo y manejo de herramientas para madera.

Con respecto a la cantidad de madera en pies/tabla que se puede secar, este secador fue diseñado para una capacidad de aproximadamente 1,200 pies/tabla, sin embargo por las características de su estructura y forro de madera contrachapada, tiene flexibilidad para crecer en forma modular a lo largo y duplicar o triplicar su capacidad, si así se desea, dependiendo de las necesidades y espacio disponible por cada usuario.

Un aspecto sin duda relevante a considerar antes de la fabricación del prototipo, es la selección del lugar en donde se colocará el producto, ya que es prioritario contar con un espacio que reciba radiación solar durante todo el día y en todas las épocas del año, para evitar que en algún período se generen sombras sobre los colectores solares, lo que disminuiría su eficiencia, ocasionando mayores tiempos de secado.

\section{FUNCIONAMIENTO DEL SECADOR}

El funcionamiento de la estufa consiste en extraer el aire caliente que se genera en el colector, con la ayuda de los dos ventiladores para introducirlo a la cámara de secado, en donde se dirige hacia las piezas de madera colocadas en el interior de la estufa, forzándolo a pasar a través de ellas. 
Con las ventilas de la secadora se puede controlar la circulación del aire en el interior de la cámara;, cuando se mantienen cerradas existe una recirculación interior debido a que el aire caliente pasa del colector hacia la madera y de la madera hacia el colector calentándose nuevamente (Fig. 4), pero si se mantienen abiertas, el aire caliente pasará del colector a la madera y después saldrá por las ventilas, inyectando así aire del medio ambiente hacia el colector (Fig. 5).

Una vez que se concluyó la construcción del prototipo, se puso a prueba todo el sistema, para saber si las partes funcionaban correctamente o si existía la necesidad de hacer alguna modificación, para así poder pasar a la etapa de prueba del secador.

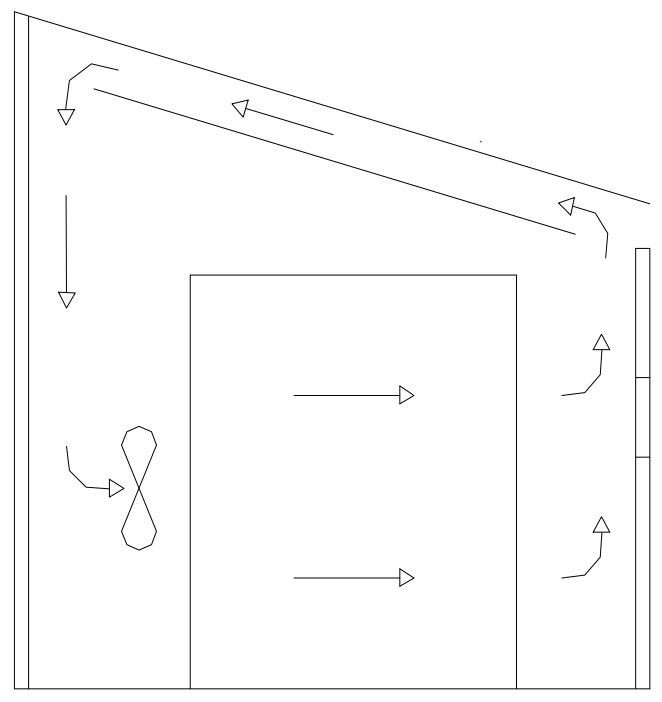

Figura 4. Funcionamiento del secador con las ventilas cerradas, para la recirculación del aire

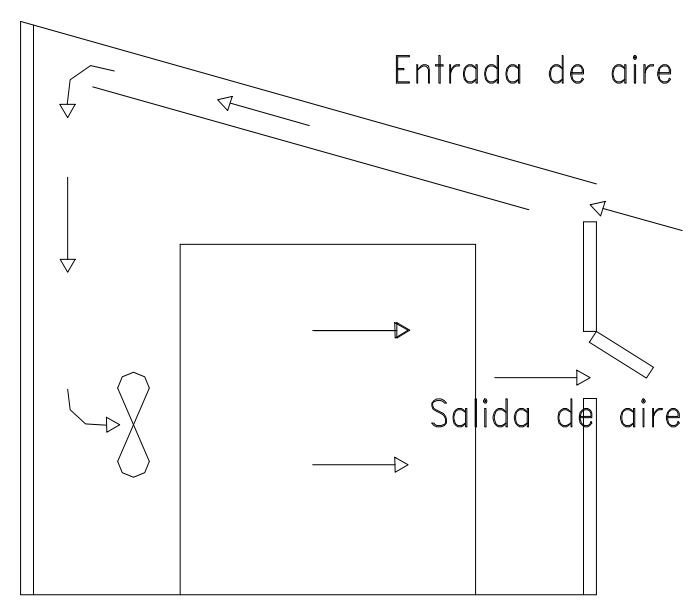

Figura 5. Funcionamiento del secador con las ventilas abiertas para la circulación del aire

\section{METODOLOGIA DE SECADO}

Al concluir la etapa de prueba y tener la certeza de que todo funcionaba en forma adecuada, se colocó la carga número 1 con madera de pino. El proceso inicia con la aplicación de cera en los extremos de las tablas que se desean secar; concluyendo esta etapa se procede a colocar la madera en el interior de la estufa, la cual se apila en la forma tradicional, colocando separadores entre cama y cama de las tablas (MartínezPinillos, 1984). Durante el proceso de colocación de la madera, se inspecciona visualmente cada una de las piezas para detectar defectos antes de someterlas al proceso; si se aprecia algún defecto, se marca para conocer su comportamiento durante el secado, repitiendo este paso con cada uno de los defectos detectados y continuando así hasta finalizar el acomodo de la madera. 
Durante esta etapa, se seleccionan cuatro tablas de entre las que tengan mayor contenido de humedad. Estas piezas se usan para saber el contenido de humedad inicial de la carga y como muestras para llevar el control diario del contenido de humedad de la madera. Dos de las muestras se colocan cerca de la entrada del aire a la madera apilada (orientada hacia el norte), la primera se coloca a una tercera parte de la altura de la madera y la segunda, a dos terceras partes de la altura; las dos muestras restantes se colocan a la salida del aire, siguiendo el mismo acomodo. El procedimiento para determinar el contenido de humedad inicial de la carga, consiste en cortar una pieza de aproximadamente $45 \mathrm{~cm}$ del centro de cada una de las tablas seleccionadas, posteriormente, de estas piezas se obtiene una porción de $25 \mathrm{~mm}$ (1 plg) de cada lado. Cuando se tienen las tres piezas se marcan y se pesan. Al valor obtenido se le denomina peso verde (PV), las dos muestras pequeñas se someten a un proceso de secado hasta extraer toda la humedad que tienen en su interior, para pesarlas nuevamente hasta tener un peso constante y así obtener un valor que se denomina peso seco (PS). Con estos dos valores se determina el contenido de humedad que cada muestra tiene al iniciar el proceso de secado, aplicando la ecuación

$$
C H=\frac{P V-P S}{P S} \times 100
$$

Posteriormente, se obtiene un valor que se denomina peso seco calculado (PSC) para cada una de las muestras grandes, usando la ecuación

$$
P S C=\frac{P V}{1+C H}
$$

donde PV es el peso verde obtenido de la muestra grande y $\mathrm{CH}$ es el valor de contenido de humedad obtenido de las muestras pequeñas, el valor de PSC calculado se usa para saber cómo disminuye el contenido de humedad de la muestra grande. Esta pieza se pesa diariamente y se aplica la ecuación

$$
\mathrm{CH}=\frac{r v-r o u}{D S r} \times 100
$$

donde PV será el peso diario que se obtenga de la muestra (Martínez-Pinillos, 1984). Con la aplicación diaria de esta fórmula, se determina el momento en que la madera llega al contenido de humedad deseado para dar por terminado el proceso de secado. Este procedimiento se sigue con cada una de las cuatro muestras seleccionadas. Al final del proceso se evalúa toda la carga, pieza por pieza, para clasificar los defectos que se pudieran haber desarrollado durante el tiempo de secado y que no estaban presentes al inicio del mismo; de esta manera se hará la evaluación final de la carga de secado.

Para llevar el manejo y control de la cámara de secado, se cuenta con medidores de temperatura y de humedad relativa, los que permiten llevar un registro de las condiciones que se tienen en el interior de la estufa durante el tiempo que dura el proceso. Esta información es útil para tomar decisiones sobre el manejo del secador y para mejorar el proceso de secado, debido a que en el secado solar la temperatura y la humedad están determinadas por la condiciones del medio ambiente en que se encuentra el secador. 


\section{CONTROL DE HUMEDAD Y TEMPERATURA}

La guía de secado básica que se usa, consiste en abrir y cerrar las ventilas a determinadas horas durante el tiempo que permanezca funcionando el secador, para controlar de esta manera la humedad relativa y temperatura en el interior. Es muy importante tener un buen control para perder la menor cantidad posible de calor (Steinmann, 1989). El procedimiento consiste en encender el sistema a las ocho o nueve de la mañana abriendo las ventilas durante media hora. Esto, con la finalidad de permitir la salida de la humedad que pudiera haberse concentrado durante el tiempo que la estufa estuvo sin funcionar; posteriormente se cierran las ventilas y se mantienen así, para que el aire circule en el interior de la secadora.

Después de este período se pueden abrir nuevamente durante media hora, alrededor del medio día para extraer el aire húmedo del interior, si se considera necesario. Durante esta etapa se debe tener especial cuidado en conocer la relación que existe entre el valor de humedad interior y el del exterior de la secadora (Steinmann, 1989) para poder decidir si es apropiado abrir las ventilas, debido a que la humedad en el exterior pudiera ser mucho más alta que en el interior.

Al concluir esto, se cierran nuevamente las ventilas y se vuelven a abrir entre las $4 \mathrm{y}$ 6 de la tarde durante media hora., al cabo de la cual se cierran y se apaga el sistema, repitiendo el procedimiento al día siguiente y los que dure el proceso de secado, hasta alcanzar el contenido de humedad deseado en la madera.

La presente es una propuesta de guía básica de secado; vale la pena mencionar que para cada carga pueden existir variaciones en los horarios propuestos y en el número de veces que se deben abrir las ventilas, puesto que se regirán por las condiciones de temperatura y humedad que se tengan en el interior y exterior de la secadora, así como por el contenido de humedad que presente la madera que se esté secando (Durand, 1984). Con la finalidad de poder llevar una mejor secuencia en el proceso de secado, el operario deberá siempre tomar en cuenta las mediciones de temperatura y humedad, para decidir los pasos a seguir durante el proceso y evitar al máximo un posible deterioro en la madera. Hay que considerar este punto como uno de los de mayor importancia, pues si el encargado del proceso de secado no tiene estos conocimientos básicos, la calidad de la madera puede disminuir notablemente.

\section{RESULTADOS}

Se muestran los resultados obtenidos con cuatro cargas de madera, en distintas épocas del año y bajo diferentes condiciones climáticas:

Carga 1. La primera carga que se sometió a prueba se hizo con madera de pino, con $25 \mathrm{~mm}$ (1 plg) de grosor y diversos anchos. Este secado se realizó en el período comprendido entre finales de mayo y principios de junio. El contenido de humedad inicial promedio de la carga era de $60 \%$.

Se mantuvo en operación el secador durante nueve días para alcanzar un contenido de humedad final de $25.4 \%$ (Fig. 6). La temperatura interior promedio que se registró durante el tiempo de secado, fue de $39.4^{\circ} \mathrm{C}$ y la temperatura exterior fue de $22.3^{\circ} \mathrm{C}$. En la figura 7 se muestra la temperatura diaria promedio que se obtuvo en el interior durante las horas que el sistema funcionó, comparándola con la registrada en el exterior y las horas diarias 
de insolación durante los días que duró la prueba. La humedad relativa promedio diaria que se tenía durante el secado, tanto interior como exterior se puede observar en la figura 8. En el interior de la secadora se trabajó con una humedad interior promedio durante los días de operación del equipo de $69.3 \%$ y la exterior fue de $47.8 \%$.

Carga 2. La segunda carga que se introdujo fue con madera de pino de $25 \mathrm{~mm}$ (1 plg) de grueso y varios anchos, el secado se realizó durante el mes de julio y el contenido de humedad inicial fue de $76 \%$.

Se obtuvo un contenido de humedad del $30 \%$ en los primeros seis días; en los siguientes cinco días se pudo llegar a un contenido de humedad de $10 \%$ y en cuatro días más alcanzó un $8 \%$ (Fig. 6). Este proceso llevó quince días; se trabajó con una temperatura interior promedio de $\begin{array}{llllll}3 & 9 & 8 & 8 & \circ & \mathrm{C}\end{array}$ y en el exterior $20.7^{\circ} \mathrm{C}$. Los valores diarios promedio registrados en el interior, durante las horas de funcionamiento de la estufa y comparados con los del exterior se pueden observar en la figura 9, así como las horas de insolación. La humedad relativa

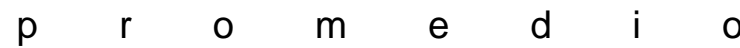
de todos los días del secado fue de $59.9 \%$ a interior de la secadora y $53 \%$ en el exterior de la secadora; en la figura 10 se pueden observar los valores diarios promedio para la humedad, tanto en el exterior como en el interior.

Carga 3. La tercera carga de secado, fue con madera de pino de $25 \mathrm{~mm}$ (1 plg) de grosor y con diferentes anchos, efectuada a finales de agosto y principios de septiembre.

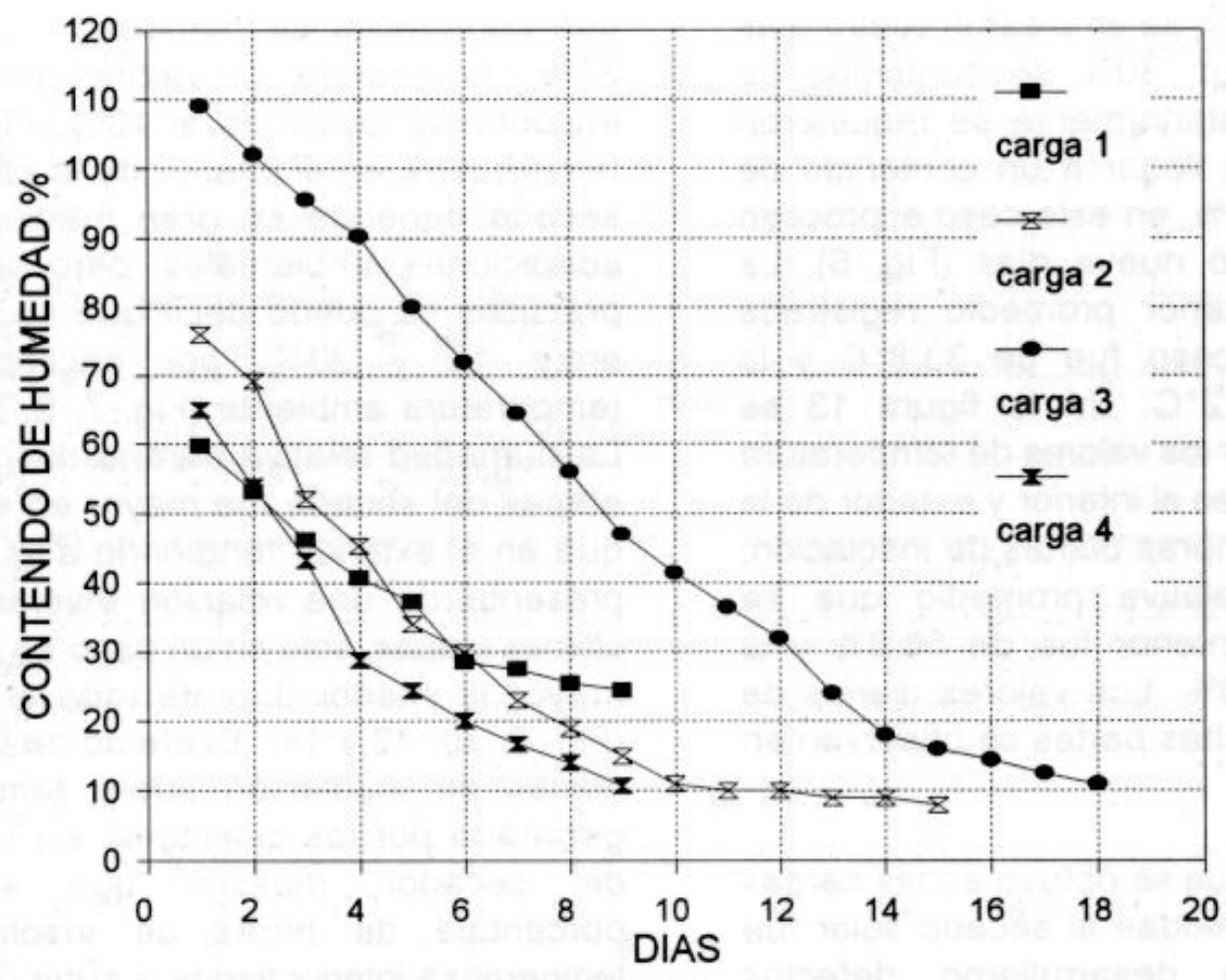

Figura 6. Variación del contenido de humedad con el tiempo en las cuatro cargas ensayadas 
Se inició el proceso de secado de la madera con un contenido de humedad de $109 \%$, logrando llegar a un contenido de humedad de $30 \%$ en aproximadamente doce días; durante los siguientes seis días se bajó el contenido de humedad hasta $11 \%$, usando un tiempo total para el secado de dieciocho días (Fig. 6). La temperatura interior promedio con la que se realizó el secado fue de $36^{\circ} \mathrm{C}$ y la registrada en el exterior de la secadora fue de $20^{\circ} \mathrm{C}$; en la figura 11 se presentan los valores promedio diarios de temperatura tanto interior como exterior y las horas diarias de insolación; la humedad relativa exterior e interior diaria promedio se muestra en la figura 12, con valores de humedad interior promedio de $64.8 \%$ y $56.7 \%$ para el exterior.

Carga 4. La cuarta carga se hizo con madera de hule (Hevea brasiliensis), a fines de noviembre y principios de diciembre, con piezas de $25 \mathrm{~mm}$ (1 plg) de grosor y diversos anchos. Al iniciar el proceso desecado se tenía un contenido de humedad de $65 \%$; se emplearon cuatro días para llegar a un $30 \%$ de contenido de humedad y posteriormente se requirieron cinco días para llegar a un contenido de humedad del $11 \%$, en este caso el proceso de secado duró nueve días (Fig. 6). La temperatura interior promedio registrada durante el proceso fue de $33.8^{\circ} \mathrm{C}$ y la exterior de $18.2^{\circ} \mathrm{C}$. En la figura 13 se pueden observar los valores de temperatura diaria promedio en el interior y exterior de la secadora y las horas diarias de insolación; la humedad relativa promedio que se registró en el interior fue de $56.8 \%$ y la exterior de $60.6 \%$. Los valores diarios de humedad en ambas partes se observan en la figura 14.

La calidad que se obtuvo en las cargas de madera sometidas al secado solar fue buena; no se desarrollaron defectos además de los detectados en las tablas al principio del secado, salvo el incremento de algunas rajaduras en los extremos de las tablas que ya existían con anterioridad al inicio del secado, las cuales aún con el sellado de los extremos no fue posible detener su avance.

Con relación a un posible testigo de secado al aire libre, no se realizó esta operación, debido a que las cargas de secado que se mencionan en este artículo corresponden a maquilas realizadas a usuarios y a que en diseños realizados con anterioridad se obtuvo una relación entre el proceso de secado solar y el realizado al aire libre; la cual, para las condiciones climáticas del sitio en donde se puso a prueba este prototipo marca una duración de entre 45 y 60 días.

\section{DISCUSION}

En las diferentes corridas de la madera secada se observa en términos generales como tiempo promedio de secado, 15 días con contenidos de humedad iniciales de $80 \%$ y finalizando con valores que pueden encontrarse cercanos al 10\% (Fig. 6). La temperatura en el interior de la cámara de secado depende en gran medida de las condiciones ambientales, pero para fines prácticos se puede decir que se mantuvo entre 15 y $20^{\circ} \mathrm{C}$ por encima de la temperatura ambiente (Fig. 7, 9, 11 y 13). La humedad relativa durante las primeras etapas del secado fue mayor en el interior que en el exterior, tendiendo a igualarse o presentando una relación inversa en las últimas etapas, sólo en un caso se mantuvo mayor el interior durante todo el proceso (Fig. 8, 10, 12 y 14). El efecto de las horas diarias de insolación sobre temperatura generada por los colectores en el interior del secador, muestra que a mayor porcentaje de horas de insolación la temperatura interior tiende a subir (Fig. 7, 9, 11 y 13). 


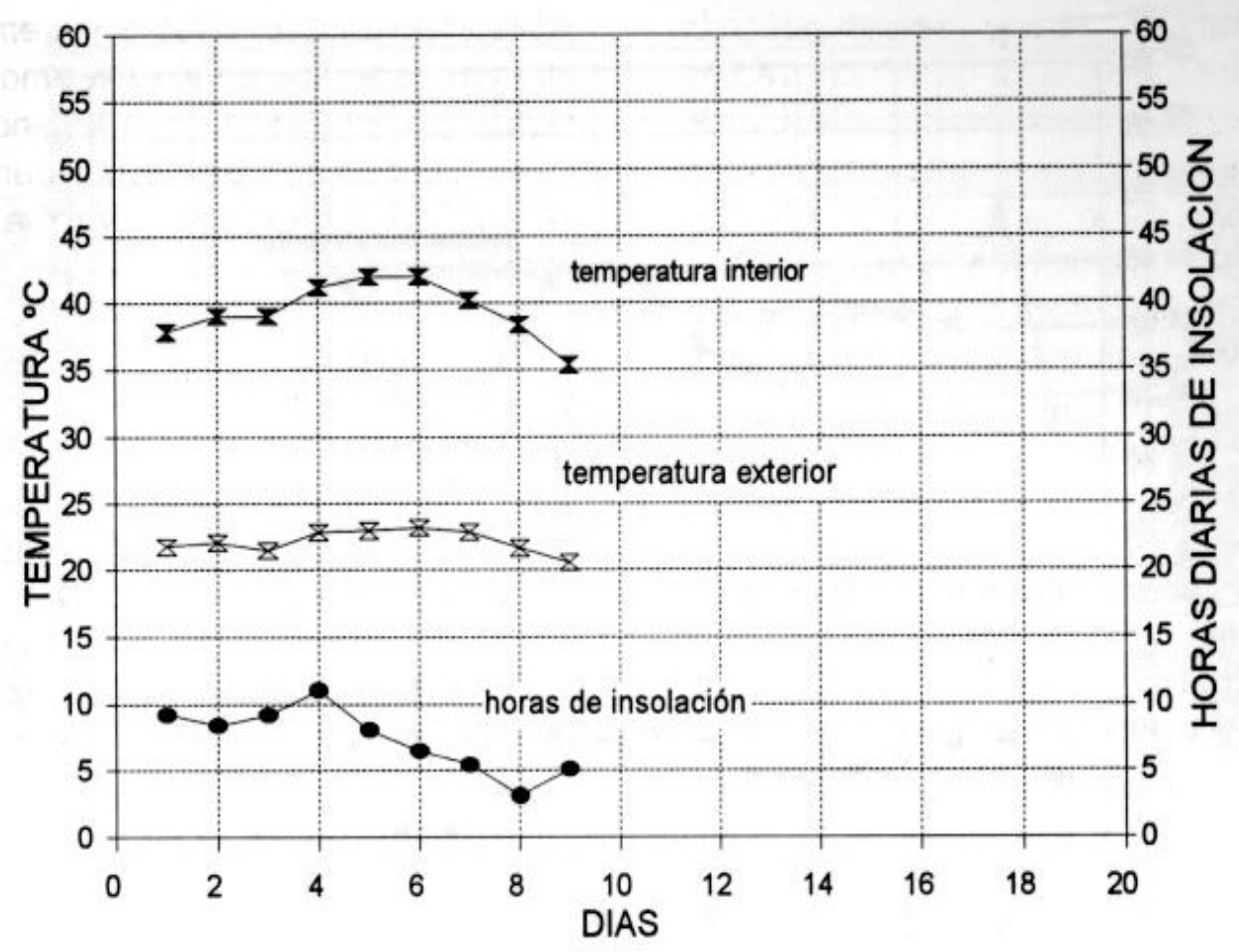

Figura 7. Temperaturas registradas en el interior y exterior del secador y horas diarias de insolación durante la carga 1 (mayo y junio, madera de pino)

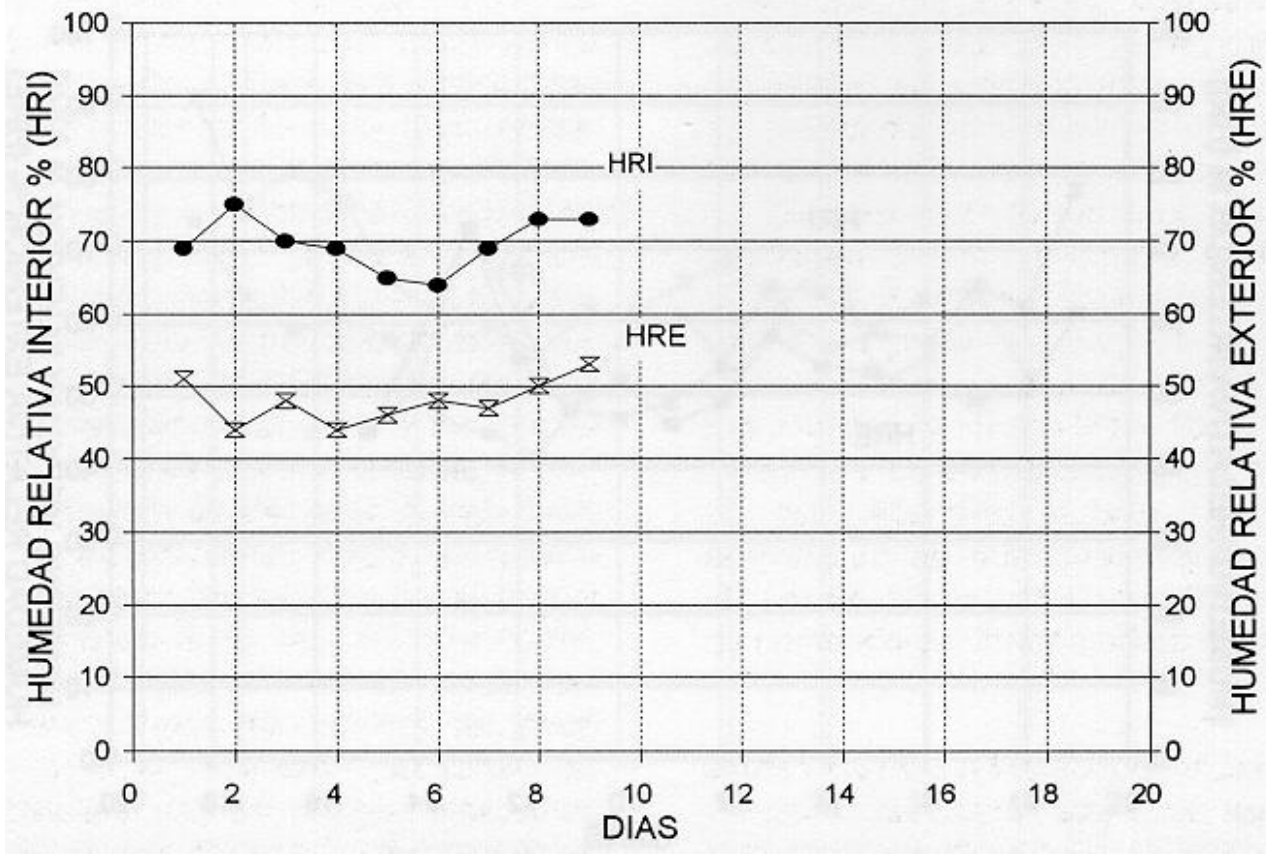

Figura 8. Humedad relativa en el interior y exterior de la estufa durante el secado de la carga 1 (mayo y junio, madera de pino) 


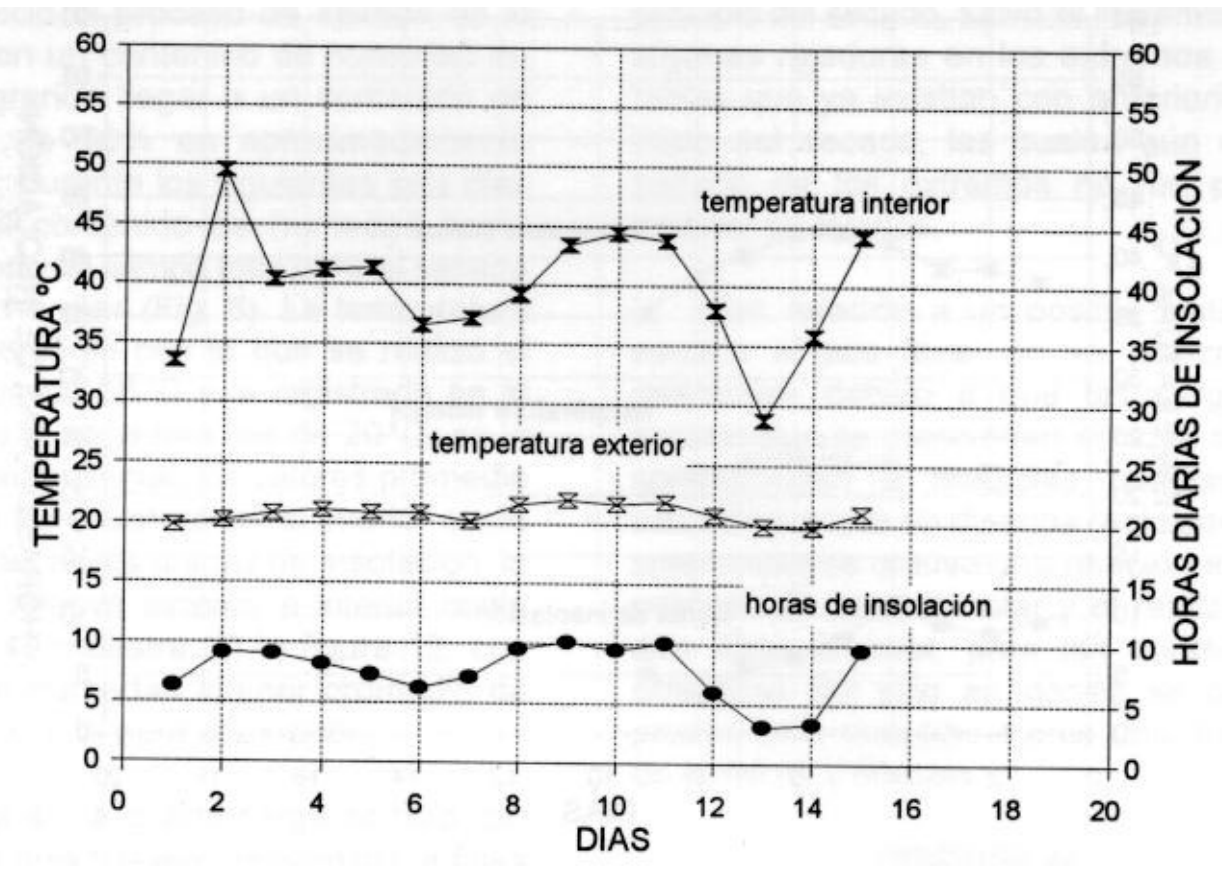

Figura 9. Temperaturas registradas en el interior y exterior del secador y horas diarias de insolación durante la carga 2 (julio, madera de pino)

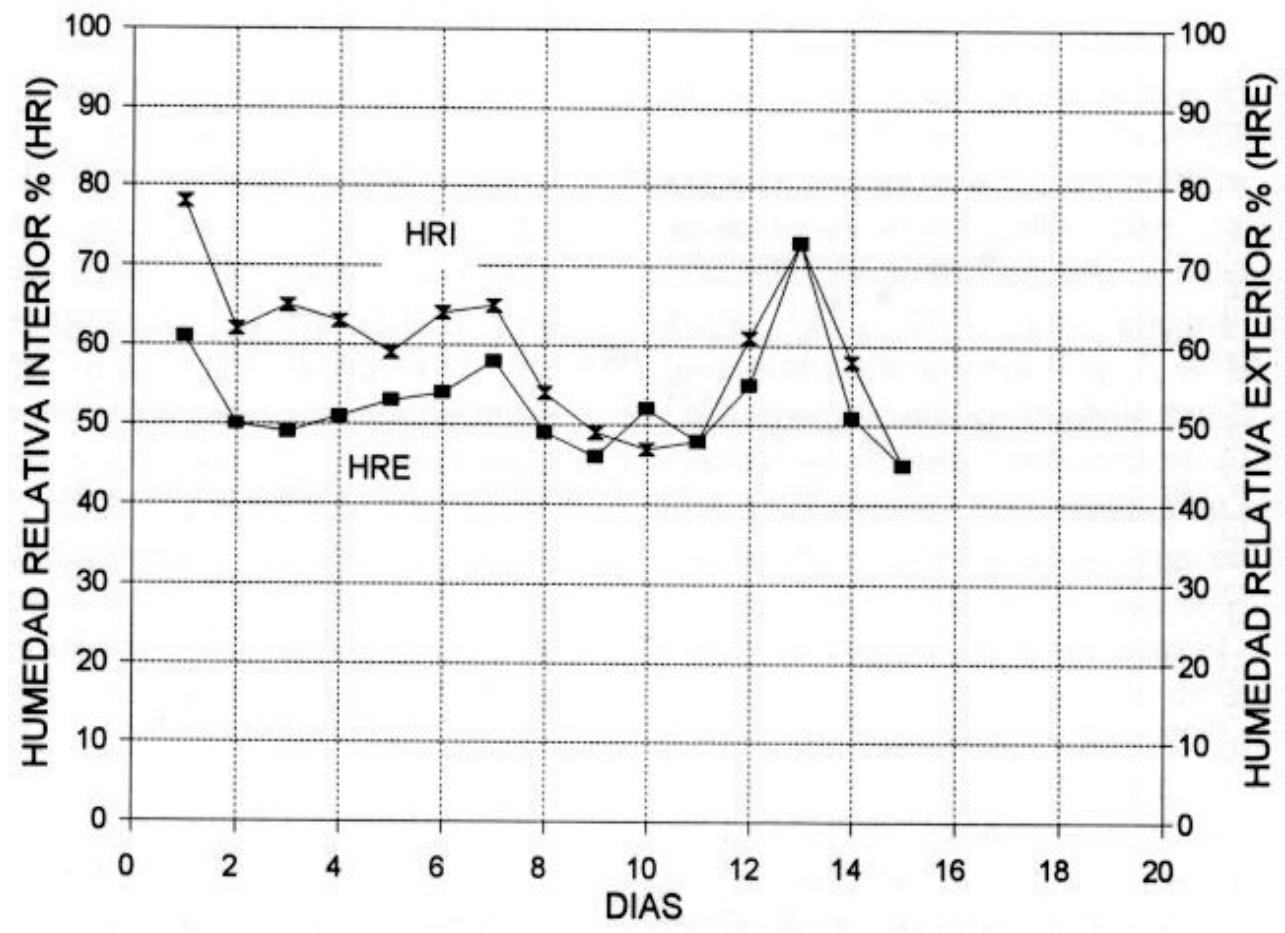

Figura 10. Valores de humedad relativa registrados en el interior y exterior del secador durante la carga 2 (julio, madera de pino) 


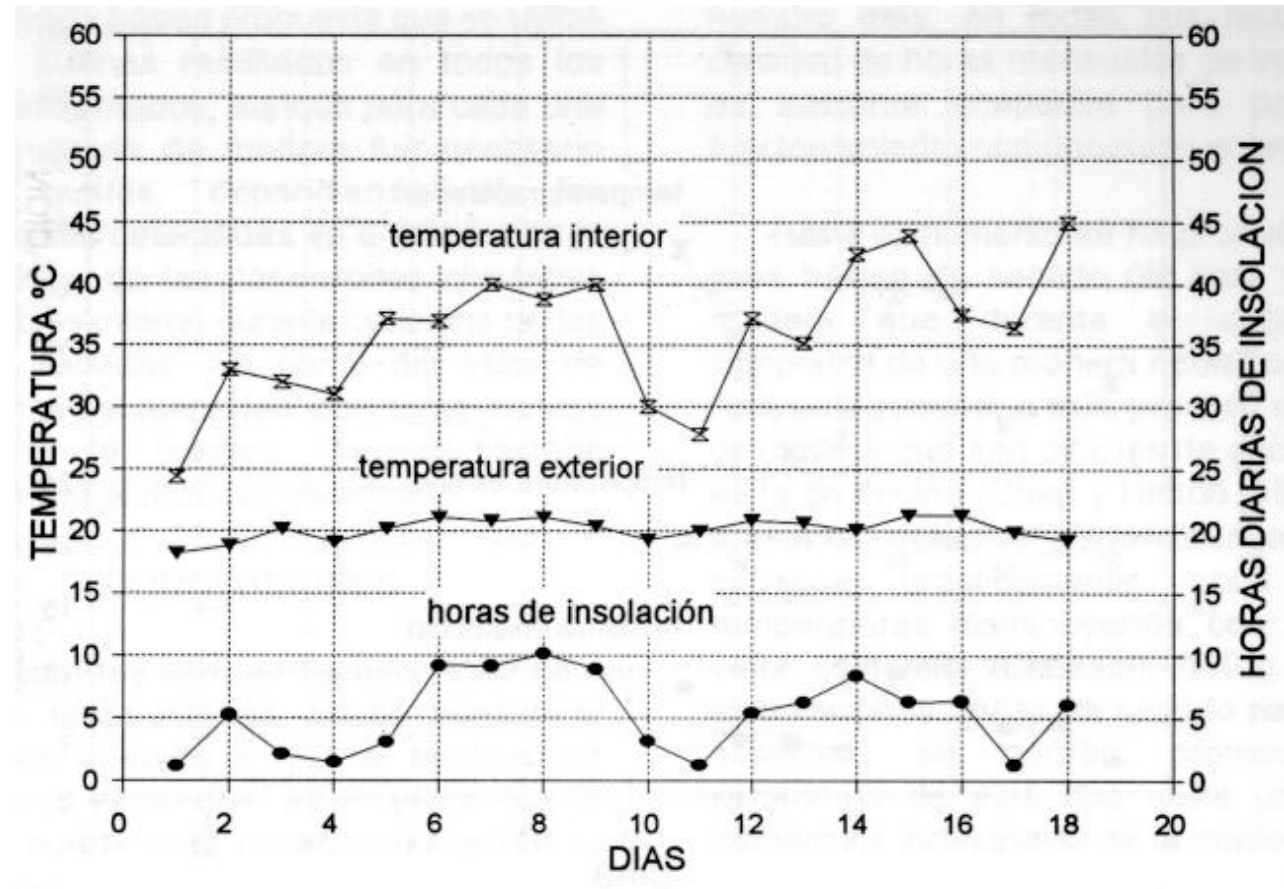

Figura 11. Temperaturas registradas en el interior y exterior del secador y horas diarias de insolación durante la carga 3 (agosto y septiembre, madera de pino)

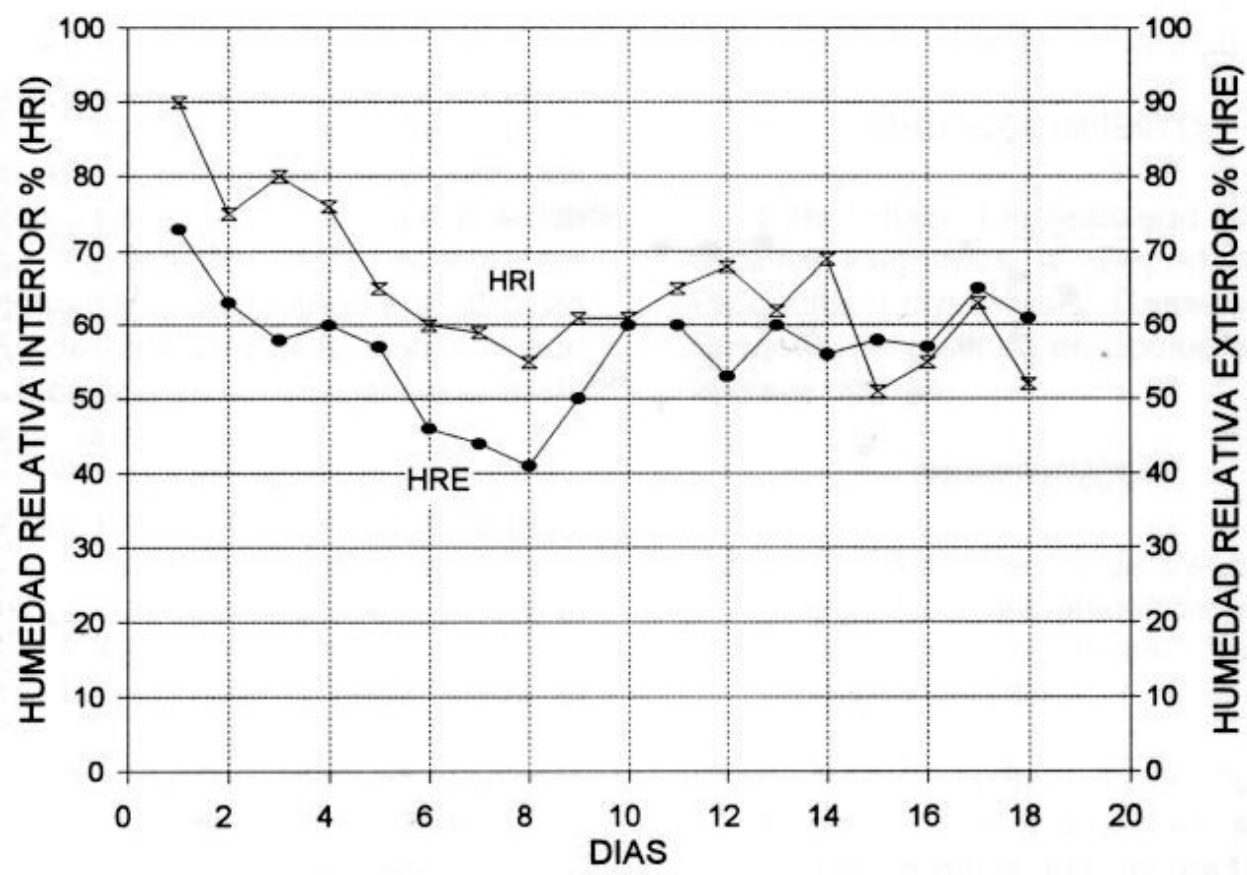

Figura 12. Humedad relativa en el interior y exterior de la estufa durante el secado de la carga 3 (agosto y septiembre, madera de pino) 


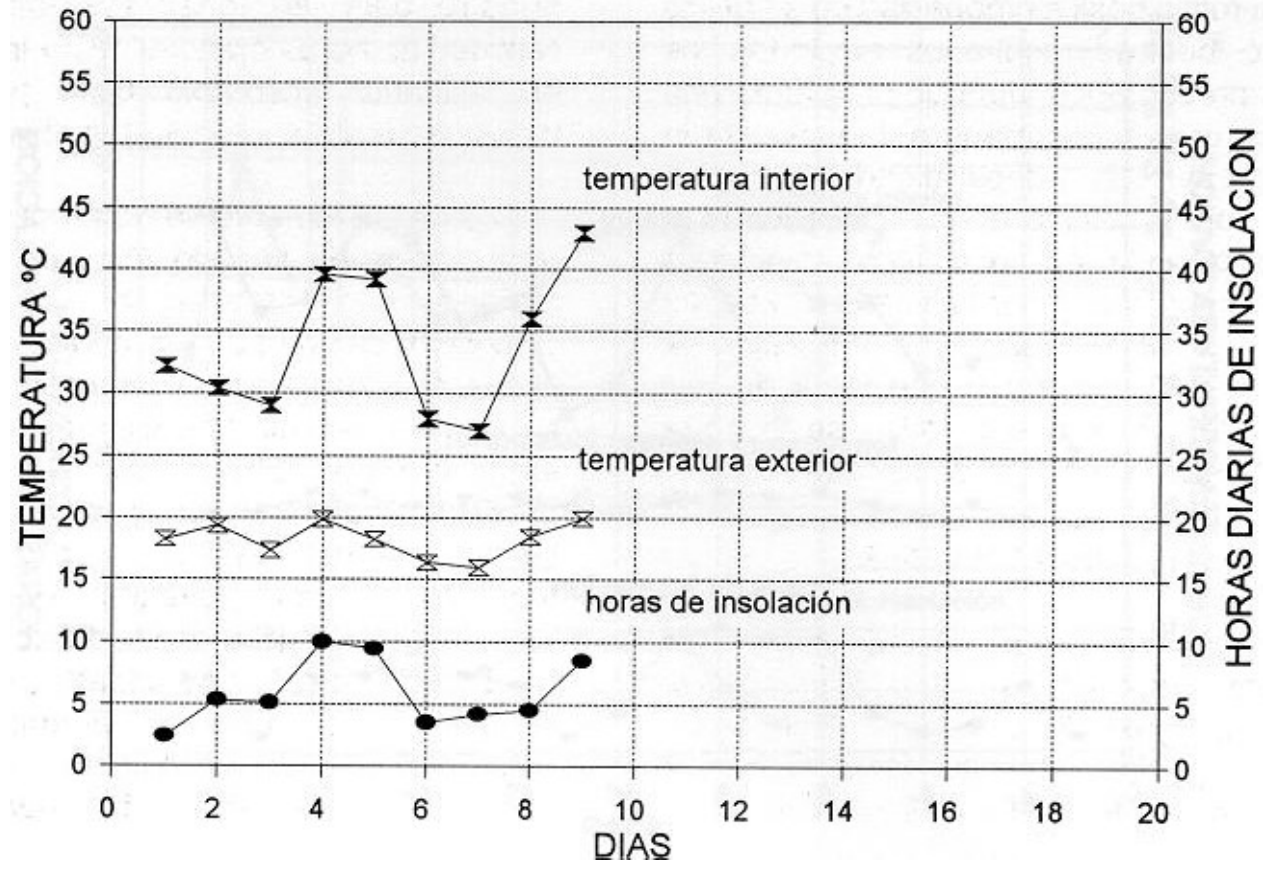

Figura 13. Temperaturas registradas en el interior y exterior del secador y horas diarias de insolación durante la carga 4 (noviembre y diciembre, madera de hule)

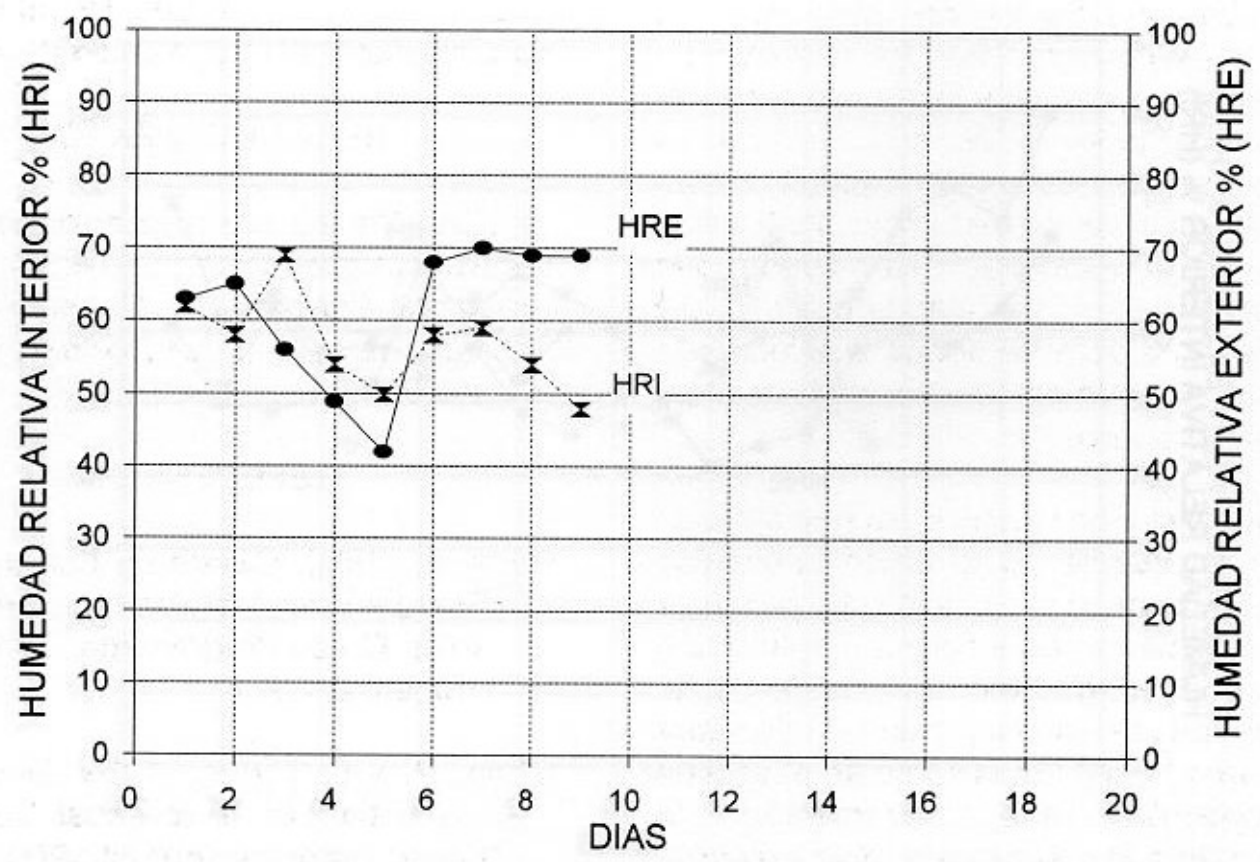

Figura 14. Humedad relativa en el interior y exterior de la estufa durante el secado de la carga 4 (noviembre y diciembre, madera de hule) 
La rutina básica propuesta que se utilizó mostró buenos resultados en todos los casos presentados, aunque para cada una de las cargas de madera fue necesario hacer ajustes, dependiendo de las condiciones detectadas en el interior de la secadora y de las condiciones climáticas que se presentaron durante cada una de las cargas secadas, así como del valor de contenido de humedad que tenía en ese momento la madera que se estaba sometiendo al proceso de secado.

\section{CONCLUSIONES}

El diseño y uso del secador solar para madera propuesto se puede considerar adecuado, debido a que al analizar los resultados obtenidos, se observa que el tiempo que emplea para secar madera es razonable.

Con relación al contenido de humedadfinal de las piezas, se obtiene madera con un mínimo de defectos y su costo en comparación con otros tipos de secadores con capacidades similares se encuentra muy por debajo, debido al menor costo energético y al valor del equipo requerido; se considera que por las características de los materiales propuestos para su fabricación, éste puede ser autoconstruible, ocasionando que el costo de mano de obra se pueda disminuir aún más, reduciendo en consecuencia el costo total del producto.

Otro factor de mucho peso que se debe tomar en cuenta es el gasto energético necesario para su funcionamiento, ya que la fuente generadora de calor es la radiación solar que es gratuita, aunque existe la posibilidad de colocar fuentes de calor auxiliares no tan caras como en el secado convencional, que incrementen la temperatura del secador y disminuyan el tiempo de secado (Simpson y Tschernitz, 1985, 1989). Por la situación geográfica de nuestro país, en todas sus latitudes la cantidad de horas mensuales de insolación es bastante aceptable para poner en funcionamiento componentes solares.

Hasta el momento se ha propuesto una guía básica de secado útil para tipos de madera que durante el secado se comportan de una manera noble, pero será necesario someter a este proceso especies de madera que son difíciles de secar como es la de encino (Chen y Helton, 1989) que presenta defectos severos cuando es secada drásticamente con altas temperaturas disminuyendo con esto su valor comercial (Simpson, 1992). Con la elaboración de guías de secado para estas especies, se podrán promover los secadores de este tipo entre un mayor número de industriales de la madera.

La forma de control con que se cuenta actualmente es totalmente manual, pero con la adaptación de controles automáticos, se puede hacer más eficiente el proceso (Steinmann et al., 1980). De ahí que se deba diseñar y adaptar equipo de este tipo.

\section{RECONOCIMIENTOS}

Este trabajo fue realizado con fondos fiscales asignados al Departamento de Productos Forestales y Conservación de Bosques, del Instituto de Ecología A.C. con la clave 902-13.

\section{REFERENCIAS}

Censolar. 1990. Centro de Estudios de la Energía Solar. Instalaciones de energía solar. Curso Programado. Tomo 2 y 5. Progensa. Sevilla.

Chen, P. y C. Helton. 1989. Design and evaluation of a low-cost solar kiln. Forest Products Journal 39(1):19-22. 
Durand, P. 1984. Experimentations with low-cost solar timber drying in Ivory Coast. North American Wood Drying Symposium. Mississippi, EUA. p:223231.

Fuentes, T. F., J. A. Silva y E. Montes R. 1996. Manual del secado técnico convencional de la madera. Universidad de Guadalajara. 122p.

Helmer, W. 1986. A general method collector sizing for solar kilns. Forest Products Journal 36(6):11-18.

Jones, C. 1983. Métodos de diseño. Gustavo Gili. México. 367p.

Martínez-Pinillos C., E. 1984. Secador solar para madera. Nota técnica No. 10, INIREB-LACITEMA. Xalapa, Ver. 15p.

Milota, M. y E. Wengert. 1995. Applied drying technology 1988 to 1993 . Forest Products Journal 45(5):33-41.

Olivera, L., C. Skaar y E. Wengert. 1982. Solar and air lumber drying during winter in Virginia. Forest Products Journal 32(12):37-44.
Rodríguez, C. E. 1988. Manual de secado de la madera. Asociación de Investigación Técnica de la Madera y el Corcho. Madrid. 140p.

Simpson, W. y J. Tschernitz. 1985. Solar wood energy dry kiln installed in Sri Lanka.. Feature article. Forest Products Laboratory. $4 p$.

Simpson, W. y J. Tschernitz. 1989. Performance of a solar wood energy kiln in tropical latitudes. Forest Products Journal 39(1):23-30.

Simpson, W. 1992. Drying technology issues in tropical countries. IUFRO Conference. Nancy, Francia. p:497-507.

Steinmann, D., H. Vermaas y B. Forrer. 1980. Solar timber drying kiln: Review of previous systems and control measures and description of an automated solar kiln. Journal of the Institute of Wood Science 8(26):254-257.

Steinmann, D. 1989. Control of equilibrium moisture in a solar kiln. Wood Drying Symposium, IUFRO. Washington, EUA. p:213-221. 